\title{
Reflets
}

Revue d'intervention sociale et communautaire

\section{Face cachée de la pauvreté au Nouveau-Brunswick : donner voix aux femmes pour comprendre la souffrance}

\section{The Hidden Face of Poverty in New Brunswick: Giving Women a Voice to Understand Their Experience of Suffering}

\author{
Isabel Lanteigne, Lise Savoie, Hélène Albert et Catherine Roy-Comeau
}

Volume 25, numéro 1, printemps 2019

Actualité de la pauvreté : débats théoriques, défis pratiques

URI : https://id.erudit.org/iderudit/1064672ar

DOI : https://doi.org/10.7202/1064672ar

Aller au sommaire du numéro

Éditeur(s)

Reflets, Revue d'intervention sociale et communautaire

ISSN

1203-4576 (imprimé)

1712-8498 (numérique)

Découvrir la revue

Citer cet article

Lanteigne, I., Savoie, L., Albert, H. \& Roy-Comeau, C. (2019). Face cachée de la pauvreté au Nouveau-Brunswick : donner voix aux femmes pour comprendre la souffrance. Reflets, 25(1), 154-175. https://doi.org/10.7202/1064672ar
Résumé de l'article

Cet article aborde la souffrance des femmes du Nouveau-Brunswick en situation de pauvreté à partir de leur expérience de vie. Leur narratif a été recueilli dans le cadre de trois recherches qualitatives. La souffrance vécue met en lumière trois dimensions soit la disqualification, la stigmatisation et les modes d'adaptation et de résistance. L'analyse de ces dimensions renvoie à la prise en compte de l'expérience subjective de la chute associée à la disqualification, de la honte liée à la stigmatisation, ainsi que de l'espoir et des luttes qui permettent de s'adapter et de résister. Ce regard propose de comprendre la pauvreté au-delà d'une perspective individualisante conviant plutôt à lutter contre la pauvreté dans une visée de dignité humaine.
Tous droits réservés (C) Reflets, Revue d'intervention sociale et communautaire, 2019
Ce document est protégé par la loi sur le droit d’auteur. L'utilisation des services d'Érudit (y compris la reproduction) est assujettie à sa politique d'utilisation que vous pouvez consulter en ligne. 


\title{
Face cachée de la pauvreté au Nouveau-Brunswick : donner voix aux femmes pour comprendre la souffrance
}

\author{
Isabel Lanteigne, \\ Professeure, École de travail social, Université de Moncton \\ Lise Savoie, \\ Professeure, École de travail social, Université de Moncton \\ Hélène Albert, \\ Professeure, École de travail social, Université de Moncton \\ Catherine Roy-Comeau, \\ Étudiante à la maîtrise, École de travail social, Université de Moncton
}

\section{Résumé}

Cet article aborde la souffrance des femmes du Nouveau-Brunswick en situation de pauvreté à partir de leur expérience de vie. Leur narratif a été recueilli dans le cadre de trois recherches qualitatives. La souffrance vécue met en lumière trois dimensions soit la disqualification, la stigmatisation et les modes d'adaptation et de résistance. L'analyse de ces dimensions renvoie à la prise en compte de l'expérience subjective de la chute associée à la disqualification, de la honte liée à la stigmatisation, ainsi que de l'espoir et des luttes qui permettent de s'adapter et de résister. Ce regard propose de comprendre la pauvreté au-delà d'une perspective individualisante conviant plutôt à lutter contre la pauvreté dans une visée de dignité humaine.

Mots clés : Femmes, pauvreté, souffrance, subjectivité, disqualification, stigmatisation 


\section{The Hidden Face of Poverty in New Brunswick: Giving Women a Voice to Understand Their Experience of Suffering}

\section{Abstract}

This article explores the suffering of New Brunswick women living in poverty through their life experience. Three qualitative research projects provided access to the narrative of these women. The suffering experienced highlights three dimensions: disqualification, stigmatization, and strategies for coping and resisting. The analysis of these dimensions emphasizes the necessity to consider the subjective experience of falling associated with disqualification, feeling ashamed as a result of stigmatization, and being hopeful and combative as strategies of resistance. Hence, these experiences of suffering reveal the necessity to understand poverty beyond an individualistic approach, and the need to combat poverty from a human dignity perspective.

Key words : Women, poverty, suffering, subjectivity, disqualification, stigmatization

Cet article veut donner la voix à des femmes francophones du Nouveau-Brunswick qui vivent diverses situations de pauvreté. Leurs discours sont tirés de trois recherches qualitatives qui portaient

1) sur la santé et la pauvreté des femmes francophones en milieu rural;

2) sur la pauvreté extrême de femmes et leur rapport aux politiques sociales de l'État (recherche toujours en cours); et

3) sur les conditions de vie des travailleuses francophones de soin à domicile en milieu rural.

Pour contextualiser la pauvreté de ces femmes, il importe de mentionner que leur revenu médian se situe à 23720 \$, comparativement à celui des hommes qui est de 35630 \$. De plus, $7 \%$ des femmes se trouvent dans une situation de pauvreté extrême, gagnant moins de 5000 \$ annuellement (Gouvernement du Nouveau-Brunswick, 2016). De surcroît, parmi la catégorie des travailleuses pauvres, les aides à domicile néo-brunswickoises gagnent un des salaires les moins élevés parmi leurs homologues canadiennes (Rose, 2014). Leur salaire horaire moyen se situe légèrement au-dessus du salaire minimum qui est fixé à 11,25 \$ au Nouveau-Brunswick (Coalition pour l'équité salariale du NouveauBrunswick, 2016). La situation de pauvreté de ces femmes prend diverses formes dans le sens où certaines d'entre elles travaillent au salaire minimum, d'autres sont en congé d'invalidité à long terme, certaines reçoivent des prestations d'assurance-emploi et enfin, 
d'autres sont prestataires d'assistance sociale. Malgré cette diversité de leur expérience, les rencontres avec ces femmes ont mis en lumière une dimension qui a été peu abordée jusqu'à présent dans nos écrits, soit la souffrance qu'entraine la pauvreté, une dimension subjective trop souvent occultée. Ce constat amène également à réfléchir à leur réalité à partir d'une analyse qui dépasse la seule privation matérielle. Dans ce qui suit, nous allons présenter l'orientation méthodologique, les perspectives théoriques sur l'expérience subjective des femmes et les résultats mettant en lumière les différentes dimensions de leur expérience de pauvreté. Enfin, nous proposerons une analyse à partir des concepts de disqualification, de stigmatisation et de modes de résistance.

\section{Orientation méthodologique}

Le présent article a comme particularité de rassembler des résultats qui émanent de trois recherches qualitatives réalisées depuis 2014 au Nouveau-Brunswick auprès de 40 femmes essentiellement francophones $(n=39 / 40)$. Alors que les participantes à ces trois recherches ont en commun la précarité de leurs conditions de vie, ce qui les singularise, c'est la spécificité de leur histoire de vie et la variabilité de leur profil individuel selon, entre autres, l'âge, les conditions de logement, la situation familiale, la situation de santé et le niveau d'éducation. Sur le plan méthodologique, ces recherches partagent aussi le fait qu'elles s'inspirent d'une perspective féministe, c'est-à-dire qu'elles placent la voix des femmes au cour de la démarche dans une visée de changement social (Ollivier et Tremblay, 2000). Deux d'entre elles ont été réalisées à partir de récits de vie (Grell, 2015), soit la première recherche portant sur la santé et la pauvreté de femmes francophones en milieu rural $(\mathrm{R} 1 / \mathrm{n}=17)$ et la deuxième portant sur la pauvreté extrême de femmes et leur rapport aux politiques sociales $(\mathrm{R} 2 / \mathrm{n}=6)$, alors que la troisième, soit celle portant sur les conditions de travail de travailleuses de soin à domicile $(\mathrm{R} 3 / \mathrm{n}=17)$ a recouru à des récits de pratique (Bertaux, 2010). Les entrevues des recherches R1 et R3 étaient d'une durée moyenne de 90 minutes, alors que celles réalisées auprès de femmes en situation de pauvreté extrême (R2), c'est-à-dire de femmes pour la plupart vivant seules et de l'aide au revenu, ne recevant que 537 \$ par mois, ont duré jusqu'à 180 minutes, tellement elles avaient besoin de se raconter. Les récits ont été étudiés à partir d'une analyse compréhensive (Kaufmann, 2006) et thématique (Paillé et Mucchielli, 2012) pour donner sens à leur expérience. Les analyses effectuées dans le cadre de ces trois études ont mis en lumière différents visages de la pauvreté, qui avaient pour commune dimension - et qui nous a particulièrement touchées — la souffrance engendrée par le fait de vivre en situation de pauvreté. 


\section{L'expérience subjective des femmes en situation de pauvreté : perspectives théoriques}

Pour bien saisir l'ancrage de cet article, il importe de présenter avant tout le sens que nous choisissons de donner à la pauvreté. Nous rejoignons les perspectives proposées par Jo (2012), Novak (1995) et Scheff (2011) quant à la pauvreté. Pour sa part, Jo (2012) reconnaît qu'au-delà du désavantage matériel et économique que la pauvreté produit, elle s'accompagne de la honte qui se manifeste à la fois " par le manque de voix des personnes qui y sont aux prises, et aussi par le manque de respect, par l'humiliation et par l'atteinte à la dignité dont elles font l'objet » (p. 516, [notre traduction]). Par ailleurs, Novak (1995) propose d'appréhender la dimension subjective de la pauvreté en référant à l'insécurité et au manque de pouvoir qu'elle génère. Cette insécurité et ce manque de pouvoir se manifestent " par une préoccupation quotidienne à savoir comment survivre à sa semaine, par le fait d'avoir peu de contrôle quant à son devenir, peu de choix, aucune occasion de planifier, pas d'espoir devant l'interminable combat pour sa survie " (Novak, 1995, p. 62, [notre traduction]). Or, le vécu de souffrance qu'occasionne la pauvreté est aussi le reflet de l'intériorisation du regard porté par l'Autre à son endroit (Scheff, 2011). Outre les contributions de Jo (2012), de Novak (1995) et de Scheff (2011) permettant de définir l'expérience subjective des femmes en situation de pauvreté, la perspective théorique féministe de Krumer-Nevo (2005) permet d'apprécier, entre autres, un type de regards pour saisir l'histoire de pauvreté des femmes. D’une part, l'auteure met en lumière la reconnaissance de la complexité et de la singularité de la vie des femmes et d'autre part, la reconnaissance de leur marginalité dans un contexte de pauvreté. La complexité et la singularité de leur vie renvoient à la diversité des expériences de vie qu'elles racontent, alors que le regard sur leur marginalité consiste à s'intéresser à la place qu' elles occupent, à l'intérieur d'un contexte social d'inégalités en raison des rapports de pouvoirs genrés. Par ailleurs, le regard social porté sur les femmes en situation de pauvreté contribue à leur stigmatisation et à leur marginalisation (Savoie et collab., 2016; Lamoureux, 2016). Souvent "autrifiées » dans un discours dominant qui tend à responsabiliser l'individu pour sa situation de pauvreté, les femmes pauvres vivent une distance sociale qui les sépare des femmes privilégiées (Savoie, Albert et Lanteigne, 2018). De plus, selon Reid et Le Drew (2013), leur expérience de pauvreté est plus intense et significative, d'autant plus qu' elles restent plus longtemps en situation de pauvreté. Or, la pauvreté qui perdure mène à l'exclusion sociale (Klein et Champagne, 2011). 
Basinski (2007), pour sa part, soulève en quoi la valeur sociale attribuée au travail marque la place occupée dans la société et le regard social posé sur l'individu. Par exemple, quand leur situation de travail se précarise par la perte d'un emploi résultant à un retrait temporaire ou permanent du marché du travail, ces femmes deviennent plus fragilisées, ayant notamment le sentiment d'être " disqualifiées ». De l'avis de Paugam (2002, p. 17), il importe de comprendre « les sentiments subjectifs de leur propre situation » en lien avec leurs expériences sociales et leurs relations avec les autres. De Gaulejac (1989) explique le lien entre l'intériorisation du regard d'autrui sur le vécu de pauvreté et le vécu de honte que ce regard génère, résultant en une perte de reconnaissance de sa propre valeur et de sa dignité, la honte faisant mal, conduisant ainsi à un vécu de souffrance. Jovelin (2017) va jusqu'à dire que cette souffrance liée à la honte et qui stigmatise conduit à l'isolement social. En parlant des travailleuses et des travailleurs pauvres, Basinski (2007) reconnait en quoi la pauvreté, dans ses dimensions sociales et politiques, a une incidence sur le vécu individuel de souffrance.

\section{Voix des femmes : l'expérience de la pauvreté racontée}

Au terme de ces trois études, l'analyse de la voix des femmes rencontrées a permis dans une démarche inductive d'entendre et de reconnaître qu' elles avaient en commun un vécu de souffrance. Ainsi, leurs récits mettent au jour les dimensions suivantes : la disqualification, la stigmatisation et les modes d'adaptation et de résistance à la pauvreté.

\section{L'expérience de disqualification comme source de souffrance}

Pour plusieurs femmes, le fait de travailler ne les met pas à l'abri de la pauvreté. En effet, avoir un emploi au salaire minimum avec peu d'avantages sociaux, comme c'est le lot de la plupart des travailleuses de soin à domicile rencontrées, leur assure un mince salaire. De surcroit, le regard que les autres portent sur elles les renvoie à leur statut précaire, dans une société où la réussite matérielle est valorisée. Le témoignage de Nicole, travailleuse de soin à domicile, reflète bien ce sentiment de disqualification :

«Si tu regardes ma famille... juste comment mes parents... j'ai un frère, il est ingénieur. Mon frère, il est scientifique. "Qu'est-ce que Nicole fait ?". "Elle travaille avec ses familles ou whatever". Comme tu le vois là... je veux dire... je ne suis pas aveugle, pis j'entends ce que le monde dit... Je me fais traiter comme si j'étais une glorified babysitter. " (Nicole, R3) 
Par ailleurs, le fait pour les femmes d'être en situation de pauvreté est souvent le résultat d'une chute à la suite d'un événement inattendu ou hors de leur contrôle, ou de plusieurs, comme recevoir le diagnostic d'une maladie (Paugam, 2002). Celles qui, sans avantages sociaux, tombent malades se retrouvent dans une situation qui les précarise davantage (Savoie et collab., 2016) en perdant de surcroît leur statut puisqu' elles ne peuvent plus travailler. Or, dans une société où les rapports sociaux sont hiérarchisés, valorisant la performance et la réussite matérielle, elles deviennent stigmatisées et marginalisées (Savoie, Albert et Lanteigne, 2016). L'histoire de Monique, 53 ans, qui est atteinte d'un cancer et qui reçoit $537 \$$ par mois d'aide au revenu, et son choix de mots illustrent bien le processus de disqualification engendré par cette chute :

"Je travaillais dans les restaurants et je me débrouillais. Je me sortais de la misère. J'avais mon char, j'avais mon chez-nous. Du manger, j'en avais. Mes factures étaient payées. Mais là, quand je suis tombée malade... Tu es toute seule. Tu es juste un être humain. Tu travailles, tu essayes de t'aider, pis là, tu tombes sur le bien-être. Tu peux plus travailler. Pis là, tu recommences à travailler, pis t'as seulement une $9^{\mathrm{e}}$ année. Pis là, asteure, une $9^{\mathrm{e}}$ année... Si je vais de nouveau à l'école, je tombe en maternelle. Je ne pouvais pas aller travailler, je me sentais tout le temps mal! J'ai été obligée d'arrêter de travailler parce que je me sentais tout le temps mal. » (Monique, R1)

Ces mots de Monique sont particulièrement significatifs dans l'image qu'elle évoque quand elle décrit son expérience en recourant au verbe "tomber». Elle perd ainsi ses statuts : elle n'est plus travailleuse, elle est assistée sociale; on ne reconnait plus sa $9^{\mathrm{e}}$ année, elle doit recommencer. Elle " tombe » malade. Elle « tombe " sur l'assistance sociale. Elle " tombe en maternelle ». C'est sa chute qu' elle décrit et la souffrance que cette chute génère.

Bien que l'État soit censé protéger ses citoyennes et ses citoyens, et que certains programmes et services viennent en aide aux personnes qui se retrouvent face à ces situations, il semble que le processus pour accéder à l'aide et la rigidité des critères ajoutent à la lourdeur de leur expérience. Ainsi, les femmes en situation de pauvreté qui ont été rencontrées font quotidiennement l'expérience d'un système qui les oblige à se justifier, à se dévoiler et à rendre des comptes, et ce, pour obtenir une aide minimale, par exemple, une boite de victuailles, ou encore un laissez-passer d'autobus. Elles sont confrontées à un système qui les surveille et qui les contrôle. Ginette, dans la trentaine, vit seule, d'aide sociale et est aux prises avec de nombreux problèmes de santé; elle témoigne de ces méthodes intrusives : 
"La première fois que je suis allée à la banque alimentaire, j’ai pu avoir de la nourriture. Ils étaient très gentils, mais il faut leur donner beaucoup d'informations. Tu dois fournir ta carte d'assurance-maladie la première fois que tu y vas, tu dois passer une entrevue, tu dois leur dire quel est le coût de ton loyer, tu dois prouver ton adresse, tu dois donner une facture d'électricité, tu dois... je veux dire, c'est très envahissant. Je ne dis pas que ce n'est pas bien, je ne dis pas que ce n'est pas nécessaire, mais c'est très envahissant. C'est très démoralisant. » (Ginette, R2)

Pour sa part Aurélia, 45 ans, reçoit également 537 \$ par mois d'assistance sociale. Elle vit dans un logement partagé subventionné. Elle raconte sa propre expérience avec la banque alimentaire en déplorant le fait qu'elle n'y ait pas accès :

«Si je vais à la food bank, je ne qualifie pas. À cause que mon loyer est juste 219 \$, je ne qualifie pas pour la food bank.»

Tout au cours de l'entrevue, cette participante — qui plutôt que de s'adresser à l'intervieweuse, se penchait vers le magnétophone pour raconter son histoire, comme pour s'assurer que ses mots soient vraiment entendus — évoque aussi les contraintes liées à l'obtention d'un laissez-passer d'autobus, elle dont le logement est éloigné des services et du centre-ville. Elle souligne :

"Je vois une psychologue qui me fait vraiment du bien, elle. Pour avoir une bus pass, il faudrait que j'aie dix appointements par mois, mais ça ne fait pas de bon sens que j'suis supposée avoir dix appointements par mois pour pouvoir être acceptée pour avoir une bus pass. C'est pas pour prendre une drive sur le bus pis aller au mall, c'est pour mon bien-être, pour ma santé mentale, c'est pour ça. " (Aurélia, R2)

Devant des situations extrêmement difficiles où un cumul de situations les disqualifie, en les faisant chuter, plusieurs femmes rencontrées ont exprimé un profond désespoir, allant pour certaines jusqu'à la contemplation de la mort volontaire comme porte de sortie. En ce sens, en raison des conditions de vie où s'entremêlent maladie, dépression et pauvreté, certaines femmes ont du mal à s'accrocher à la vie, comme en témoigne cet extrait des propos de Maria, 55 ans, une travailleuse pauvre œuvrant dans les soins à domicile et vivant avec des problèmes de santé. Elle exprime ainsi sa souffrance :

"Je pense des fois, comme je ne pense pas à mourir, mais... est-ce que c'est seulement dans ce temps-là que je pourrais dormir et dire je suis en paix? 
J'n'aurais même plus besoin d'y penser. Je ne devrais pas dire ça, mais je le dis parce que c'est ce que je pense parfois. Mais je ne veux pas mourir. J'ai peur de mourir! J'ai peur de tout on dirait. » (Maria, R1)

Au-delà de sa situation de pauvreté, Ghislaine était aux prises avec une maladie qui lui occasionnait beaucoup de douleur, et elle était aussi victime de violence psychologique et physique de la part de son conjoint. Elle raconte un moment où elle a sérieusement considéré s'enlever la vie :

«Tu sais, j'avais l'idée d'aller me suicider. J'étais assise sur mon sofa, pis là je pensais à aller me suicider dans ma chambre. J'étais dans une espèce de transe, j'étais pognée sur cette idée. J'étais tannée, j'en avais assez. » (Ghislaine, R1)

Heureusement, c'est un bruit de la voisine dans le couloir qui l'a fait sortir de ce qu'elle décrit comme une transe et qui l'a sauvée. En route vers son domicile après s'être déplacée pour recevoir un énième traitement contre le cancer qu' elle combattait, Monique aussi a eu des pensées suicidaires :

" Je pensais au suicide. Je pensais, si je vais vers le transport (camion-remorque) et que je me lance dessus, lui, à la vitesse qu’il s'en vient là... Si je survis à ça... " (Monique, R1)

C'est le fait de n'avoir rien, comme elle le dit, de vivre aussi ce qu'elle qualifiait d'enfer, qui l'amenait à avoir à plus d'une reprise ce genre d'idées. C'est par ailleurs à la pensée de sa fille qu' elle se ressaisissait, et qu'elle s'accrochait à la vie. Pour plusieurs, les enfants ont été la force qui les a maintenues en vie, comme c'est aussi le cas pour Léa :

"C'est sûr que j'ai quand même pensé à me suicider, mais je ne l'aurais jamais fait parce que c'est les enfants qui m’ont tenue là. " (Léa, R1)

Francine a raconté sa tentative de suicide à l'aide de médicaments :

«Ben, j'avais fait une overdose, $\mathrm{j}$ 'avais fait une tentative de suicide. Je ne veux pas en parler (pleure)... Je suis mieux là, mais je ne veux pas en parler. » (Francine, R1)

Ces mots traduisent la souffrance et le mal-être qui habitent ces femmes. Le tropplein de ce mal de vivre les conduit parfois à penser à l'irrévocable pour y mettre fin. C'est souvent par l'intervention de personnes significatives, par leur geste, ou encore à la seule pensée des personnes qu'elles aiment, par exemple leurs enfants, ou encore en 
imaginant la blessure qu' elles infligeraient à d'autres, qu'elles repoussent l'idée du suicide et qu'elles choisissent, malgré tout, la vie.

Ainsi, le désespoir constitue un marqueur important de la souffrance. En fait, comment est-il possible de penser pouvoir s'en sortir quand des difficultés de vie s'enchainent et que l'adversité laisse peu de place au répit, et quand, en plus, cela perdure. Les propos d'Aurélia sont éloquents en ce sens :

"Comme moi, j’suis rendue à un point, à 52 ans, où j'me dis : Ok! si je vis même jusqu'à 90 ans, c'est-tu cecite qui va être le restant de mes jours, quoi? " (Aurélia, R2)

Dans un autre ordre d'idées, la disqualification de ces femmes passe par leur manque de pouvoir qui se traduit par des choix limités et par un manque de voix. Ginette exprime son absence de choix :

«Mais la plus grosse affaire dans ma situation, c'est qu'il n'y a pas de choix. Je ne peux pas simplement appeler un ami et sortir pour prendre un café, tu sais. Quand je suis à la banque alimentaire, je ne peux pas dire, vous savez : " Je veux du fromage aujourd'hui ». Il n'y a pas de choix. J'ai si peu de choses que je peux contrôler. (Pleure) Je ne peux pas contrôler la météo, je ne peux pas contrôler les choses qui se brisent dans mon appartement, j'ai peur de devoir payer des factures. Je ne peux pas contrôler... Je ne peux même pas tomber malade parce que je n'ai pas les moyens de me payer des vitamines... Je n'ai pas les moyens de payer! Une des raisons pour lesquelles j’ai été si malade, c'est que je ne pouvais pas aller dans une clinique, parce que je n'avais pas l'argent du bus pour aller à une clinique. Je n'ai juste pas de choix! Vous savez, c'est tellement difficile, et ça vous épuise. » (Ginette, R2)

Ce dernier témoignage illustre comment le manque de choix agit sur la vie des personnes.

Ginette craint les imprévus puisque ceux-ci viendraient aggraver sa situation et elle n'a pas les moyens d'y faire face. Elle se sent dépourvue et sans contrôle sur sa situation. Une tempête de neige, par exemple, amplifie les obstacles qu'elle doit surmonter en raison de son état de santé pour se rendre à un rendez-vous médical. Cette femme qui utilise une petite chaufferette électrique a raconté avoir contracté une pneumonie parce qu'elle n'avait pas les moyens de chauffer adéquatement sa demeure. Comment se surprendre qu'elle soit si préoccupée par la météo? 


\section{L'expérience de stigmatisation qui génère de la honte}

En racontant leurs trajectoires de vie, plusieurs des femmes rencontrées parlent de leur expérience de stigmatisation, de la souffrance et de la honte ressenties à divers moments de leur vie, sans nécessairement les nommer ainsi. Dans leur recherche, Chase et Walker (2012) soulignent en quoi les personnes en situation de pauvreté utilisent une variété de mots pour traduire le vécu de honte. Les femmes rencontrées dans le cadre de nos recherches ont fait allusion aux expériences suivantes : entre autres, se sentir dégradées, se sentir basses, faire l'objet de moqueries, se sentir gênées. Cette expérience de honte part souvent de situations qui remontent à l'enfance où elles ont fait l'objet d'étiquetage et d'intimidation en raison de leur pauvreté. De Gaulejac (1989, p.130) rappelle que « c'est le mépris de l'autre qui engendre la honte ». Par exemple, Jeanne (R1) raconte comment le fait d'avoir été pauvre durant l'enfance et d'aller à l'école sans le matériel nécessaire ni les vêtements à la mode a fait en sorte qu'elle a subi de l'intimidation, ce qui a affecté son parcours scolaire. D'autres femmes, dont Katherine, ont quitté l'école en raison du vécu de honte exacerbé par l'intimidation :

"Les autres disaient tout le temps que j'étais grosse pis laide, pis ils se moquaient tout le temps de moi à l'école. Ils m'appelaient tout le temps une grosse plug. Ça a commencé quand j'étais en grade 4, peut-être. Pis là, ils me mettaient de la gomme dans les cheveux. Une fois, j'avais de la gomme dessous mes cheveux. Il a fallu que ma mère me coupe une grosse poignée de cheveux (silence). » (Katherine, R1)

Ces expériences sources de honte qui sont présentes à plusieurs moments de leur trajectoire se manifestent également dans diverses sphères de leur vie, à savoir la vie personnelle, familiale, professionnelle ou sociale. En effet, ces diverses sphères risquent d'être affectées, entre autres, selon le type de logement occupé, l'endroit où est situé leur logement, le type de travail qu'elles occupent, ou encore le fait de recevoir de l'assistance sociale. La situation de Maria illustre en quoi le fait de vivre en logement subventionné par l'État est source de stigmatisation et de honte, non seulement pour elle, mais aussi pour son fils :

" J'avais commencé à travailler au restaurant. J'avais déménagé chez mon père, pis il fallait que j'applique pour un NB Housing [logement subventionné]. Mon garçon n'était pas trop content qu’on déménage là. Il m’a dit: "Mame, on déménage sur la rue des pauvres". Pis j’ai dit : "Où est-ce que tu veux qu'on déménage? Moi je ne peux pas payer un vrai appartement. Je ne peux pas payer 500 \$, plus électricité, plus tout ça par mois. » (Maria, R1) 
Toujours en lien avec le logement, Léa raconte comment elle se sent face au regard que posent les autres sur elle :

" Je me sentais vraiment basse, je me sentais vraiment basse à cause du monde. C'est de même qu' ils te font sentir. Je restais dans un NB Housing, pis il fallait j'aille payer mon loyer. Là, on avait notre petit livre, il fallait qu'on amène notre petit livre à la Caisse. J'avais juste roulé des rouleaux de 25 cents pis d'une piasse [pièce d'un dollar]. La caissière m'a dit : "Ah! Bien, nous autres on ne peut pas accepter ça”. J'ai blowé [fait une crise], j’n'ai pas payé mon loyer. J'suis sortie de là, non, non! J'étais enragée parce que je sentais que parce que j'étais sur le welfare, ils me regardaient différemment. » (Léa, R1)

Pour certaines femmes, et c'est le cas d'Hélène, l'emploi occupé et la comparaison avec les personnes de son entourage produisent un sentiment de honte :

"J'avais honte de travailler dans une usine. Ah oui! Ah oui. C'était comme dégradant. C'est que tes chums avaient tous gradué et puis toi, tu travaillais dans une usine à poisson. Mais quand j'suis tombée sur l'aide au revenu, là, j'suis tombée encore, c'était plus dégradant. Et après j'n'ai jamais eu honte de travailler dans une usine... » (Hélène, R1)

Bien que son travail dans une usine à poisson ait provoqué chez elle de la stigmatisation et de la honte, le fait de recevoir de l'assistance sociale en génère davantage. Carmelle exprime aussi clairement sa honte d'être prestataire d'assistance sociale :

"On dirait que je ne pouvais pas accepter ça. Je disais tout le temps à la femme [la fonctionnaire], à chaque mois : "Aussitôt que je vais être correcte, je vais aller travailler. Moi, je ne veux pas être... Je ne veux pas prendre ça tout le temps". Quand j'ai été lui dire, au bout d'un an, que je commençais à travailler, elle a dit: "Je savais que toi tu ne resterais pas longtemps là-dessus, t'étais bien qu'trop fière." (Carmelle, R1)

La réaction de cette fonctionnaire semble refléter un des regards posés par la société à l'endroit des prestataires d'assistance sociale à savoir l'absence de volonté de s'en sortir, et ce, par manque de fierté.

De plus, être un parent en situation de pauvreté génère un vécu de honte et soulève des inquiétudes quant au regard possiblement posé sur son enfant. Délia raconte la honte qu' elle ressent lorsqu' elle envoie son enfant à l'école avec presque rien pour son dîner : 
«Je n’ai jamais lâché pour l'amour de mes enfants, mais j’ai eu honte, j’ai eu très honte. » (Délia, R1)

Quant à Hélène, sa gêne liée à ses conditions de vie fait en sorte qu'elle choisit de se priver pour offrir à sa fille de beaux vêtements, pour cacher un tant soit peu sa pauvreté et ainsi, ne pas exposer son enfant à la honte :

"Je ne voulais pas que le monde sache que sa maman était sur l'aide au revenu. " (Hélène, R1)

Bélinda éprouve une grande préoccupation quant à la stigmatisation dont son fils pourrait faire l'objet. Pour elle, la rentrée scolaire est particulièrement difficile. Elle doit dépendre de sa carte de crédit pour faire les achats susceptibles de protéger son enfant de la honte :

«En septembre, quand le petit retourne à l'école, ce n'est pas vrai que j'ai une couple de 100 \$ pour l'habiller. Ça fait que le petit peu que j'ai pu ramasser en économisant icitte pis là, bien je vais le prendre pour ça. Mais la balance là, ça, c'est la carte de crédit. Pis là, je veux dire, il faut qu’il soit habillé comme les autres là. Je ne peux pas dire : "Non, non, toi tu n'as pas le droit d'être habillé comme ça". Parce que c'est déjà catégorisé en maternelle là, en rentrant à l'école, là! Je veux dire, soit t'es pauvre, t'es moyen ou t'es riche. Ça fait qu'il n'est pas pauvre, il n'est pas riche, mais je veux qu'il soit dans la normale. Je veux qu’il passe en général. " (Bélinda, R1)

Dans sa vie sociale, Ginette éprouve souvent de l'embarras en raison de sa pauvreté. Elle refuse à l'occasion des invitations ou elle a recours à des stratégies pour dissimuler sa pauvreté :

"Alors, je veux dire, c'est vraiment... C'est très isolant. Et c'est embarrassant. J'ai des amis qui m'invitent pour le thé, le café, et à beaucoup d'endroits ... j'essaie de prendre une tasse d'eau chaude et de prendre un sachet de thé que j'ai amené de la maison sans que personne ne s'en aperçoive. Mais, je dis aussi "non" souvent. Les gens ne m’invitent plus à des endroits parce que je dis "non" souvent, mais ce n'est pas toujours parce que je veux dire "non", mais c'est souvent parce que je ne peux pas!» (Ginette, R2)

Certaines femmes ne veulent pas dépendre de leur entourage et conséquemment, elles demandent très peu d'aide. Bien qu'elle ait besoin d'argent, Ghislaine n'oserait jamais en demander : 
"Bien moi, je suis habituée de même. Mon garçon, jamais je ne lui demanderais un cent. » (Ghislaine R1)

La honte ressentie en raison de sa situation de pauvreté est en quelque sorte masquée par la fierté et le besoin de préserver sa dignité. Quant à Bélinda, conserver sa dignité repose sur sa manière de trouver dans sa vie des sources de fierté. Son autonomie en est un exemple :

"Je suis consciente de qu'est-ce que j'ai et je suis fière de qu'est-ce que j'ai accompli. Je veux dire, ce n'est pas énorme, mais je l'ai fait moi-même.» (Bélinda, R1)

Elle ajoute qu'elle ne veut surtout pas que les gens aient pitié d'elle. Ainsi, pour de nombreuses femmes comme Monique, il est important de maintenir sa dignité :

"Regarde, je suis pauvre, mais j’ai ma fierté pareille, ma fierté pour moi.» (Monique, R1)

En somme, les expériences de souffrance et de honte évoquées, bien qu'intériorisées, proviennent plutôt de regards, de gestes et de systèmes sociaux qui méprisent, qui disqualifient et qui stigmatisent.

\section{Espoir et lutte comme modes d'adaptation et de résistance à la pauvreté}

Les récits des femmes rencontrées dans le cadre de nos recherches dans le présent article mettent en lumière leur espoir qui est variable en fonction, entre autres, des aléas de la vie. L'espoir observé dans leur narration se manifeste par les rêves qu'elles entretiennent et les objectifs qu'elles se fixent. Pour certaines, comme Aurélia, ce rêve vise à récupérer son appartement et ainsi son espace afin de s'adonner à ses passe-temps et avoir un minimum de qualité de vie:

"C'est mon rêve d'avoir mon appartement de nouveau, tu sais. J'ai besoin j'ai une guitare dans ma chambre, j'veux prendre des leçons de guitare, tu sais. J'veux mon espace, mon espace. J'aime beaucoup faire de l'artisanat, mais je n'ai pas de place, tu sais. " (Aurélia, R2)

De son côté, Jeanne rêve de travailler un jour en milieu hospitalier et se fixe des objectifs pour y arriver :

«Mon prochain but, c'est d'avoir ma $12^{\mathrm{e}}$ année. » (Jeanne, R1) 
Rose, quant à elle, aimerait bien suivre une formation :

"Je n'ai jamais été suivre de cours, mais j'aimerais ça y aller. C’est pour ça qu'asteure, je veux essayer d'agrandir mon horizon pour me trouver des objectifs. Tu sais, essayer d'aller de l'avant. Je vais bien pareil. J'ai accompli pas mal pareil dans ma vie, dans mes années toutes seules. J'ai encore un petit chez nous. » (Rose, R1)

Nina, pour sa part, rêve d'écrire un livre pour exprimer ses états d'âme :

" J'aime écrire, j’ai déjà plein, plein de poèmes que j'aimerais à un moment donné mettre ensemble et faire un livre. Mais, je n’ai jamais eu le temps... Écrire, c'est une manière pour moi de sortir ce qui est dans moi, pis le partager à d'autres. Éventuellement, si je peux avoir du temps, pis me retirer avant que je sois morte (rire), je veux essayer de tout mettre ça ensemble. " (Nina, R1)

Enfin, Paula (R1), dans la vingtaine, rêve d'être travailleuse sociale et de voyager. Elle souhaite une vie meilleure comme celle de ses amies qui ont une carrière. Pour plusieurs des participantes, ces aspirations, ces rêves, ces espoirs sont à l'origine de leur besoin d'avoir confiance dans la vie, de s'y accrocher, et d'espérer une vie meilleure, plus sereine, plus harmonieuse, plus douce. L'espoir caressé semble alléger la lourdeur de certaines situations difficiles de leur vie.

Dans un autre ordre d'idée, le fait d'avoir à interagir avec les agents de l'aide au revenu met aussi en lumière leur pouvoir limité, ce qui constitue une source d'indignation pour certaines d'entre elles. Édith, 60 ans, reçoit de l'aide au revenu. Elle partage son indignation devant les difficultés qu'elle rencontre quand elle doit demander des services :

"Je ne comprends pas qu'est-ce que ça veut dire, le bien-être social. Quand une personne est dans la misère, pourquoi ils n'aident pas! Je ne comprends pas encore... Moi, j'ai vu une madame pis elle, ses piqures étaient payées. Pis moi, elles n'étaient pas payées. J'me suis dit : "Pourquoi ils font ça?" Il n'y a pas de justice, on dirait. Je ne me défends plus! Pourquoi me défendre? Je sais que je ne gagnerai pas. L'autre, le petit, pis l'autre, le haut qui a des gros salaires, lui par exemple, le gouvernement peut les aider à coup de millions pis de 500000 \$. Bien, le petit, lui, crève. Arrange-toi avec ton petit 537 \$, pis regarde, c'n'est pas notre problème à nous autres. Je trouve ça injuste, injuste, incroyable! » (Édith, R1) 
Ce témoignage d'Édith reflète comment il est difficile de se faire entendre et de revendiquer pour de l'aide afin d'améliorer ses conditions de vie. La situation de Marielle, 58 ans, dont le seul revenu familial consiste à la faible pension d'invalidité de son conjoint, est également révélatrice de cette difficulté à négocier avec le système relatant le manque de pouvoir et la nécessité de lutter. Son mari et elle ont travaillé au salaire minimum une grande partie de leur vie. Mais en raison de problèmes de santé, ils se retrouvent aujourd'hui dans une situation précaire, leur maigre revenu ne leur permettant pas de payer les médicaments nécessaires :

"Les médicaments, ça me met dans le trouble à tous les mois, moi j'ai deux prescriptions et mon homme en à dix. " (Marielle, R2)

Pilier de la famille, Marielle tente par tous les moyens de trouver des solutions qui leur permettraient de survivre. Elle raconte les défis auxquels elle est confrontée lors de demandes adressées aux agentes ou agents du système :

"J'ai commencé à être pauvre pis j'essayais d'avoir du welfare, et le welfare ne voulait pas maider parce que mon homme a une pension. Maintenant, je suis après me battre pour la carte blanche [carte d'assurance médicaments de l'aide au revenu] pour mon homme. Comme ça, il pourra aller se faire arracher ses dents. Il a cinq dents qui sont pourries dans sa bouche. Pis des lunettes, il devrait avoir des lunettes, il est diabétique. Tu sais quoi, ça fait un an que je me bats avec eux! Ils disent que j'ai trop d'argent. À toutes les fois que je téléphone, ils veulent savoir tout ce que je fais et je leur dis, mais je n’ai pas de réponse. Par exemple, il y a deux semaines je leur ai envoyé tous mes papiers, les factures et tout. Ils ont téléphoné pour dire que ce n'était pas assez, ils en veulent d'autres. J'ai amené ce qu'il demandait et ils n'ont pas retéléphoné. Quand je vois ça, ça me brise parce que moi je me bats pour des médicaments, juste ça! T'as pas besoin de me les donner à moi, juste pour mon homme, you know! Vous ne voulez pas me donner ma carte blanche, donnez-la à mon homme, c'est lui qui en a de besoin. » (Marielle, R2)

Lors de la rencontre, Marielle a qualifié ses interactions avec les agentes ou agents de l'État de "sa bataille avec le welfare».

Même si les femmes travaillent, leurs conditions d'emploi sont souvent si précaires qu'elles risquent de se retrouver sans revenu si, par exemple, elles tombent malades. Manon, 52 ans, est travailleuse de soin à domicile. Elle raconte : 
« Non, je n'ai rien du tout, c'est ça que je trouve dur. Si tu vois un docteur pis qu' il te met off parce que tu as une grippe et qu'elle va durer une semaine, bien qu'est-ce que tu fais? Tu n'as pas de paie, tu n'as pas de paie. » (Manon, R3)

Cette situation est particulièrement angoissante pour Manon qui est l'unique pourvoyeuse de sa famille puisque son mari est malade. Les femmes qui comme Manon travaillent dans les soins à domicile le font de façon isolée et sans protection sociale. De crainte de perdre leur emploi, elles ont peur de revendiquer de meilleures conditions de travail.

Par ailleurs, un système qui surveille et contrôle à ce point peut engendrer des comportements de subversion. Tout en se distanciant des comportements subversifs qu'elle évoque, c'est l'analyse qu'en fait Ginette, 35 ans, qui vit de l'aide au revenu :

"Tout est caché. Tout est un combat, tout est caché... Et honnêtement, ce que je déteste du système, c'est que j'ai l'impression que ça nous force à faire — et je ne l'ai pas fait — que ça nous oblige à être sournois, ou à tricher, ou mentir, ou voler, ou quelque chose, parce que nous, on ne peut pas arriver. » (Ginette, R2)

Si certaines femmes sont contraintes à contourner le système pour survivre, d'autres s'adonnent à des activités de revendication dans une visée de changement social. Par exemple, Caroline, 67 ans, travailleuse de soin à domicile à temps partiel raconte :

"Le gestionnaire de cas demandait tellement de questions pis il te harcelait pis il venait te voir. Les fois que c'est arrivé... j’me suis dit : "Comment estce qu'on est traitées? ” C'est pour ça que j'ai embarqué sur le comité pour la justice sociale, parce que je sais ce que c'est, je les défends, parce que le gouvernement, c'est incroyable... c'est incroyable... chez les gens aussi, les préjugés, tu sais... J'ai eu du bien-être à 60 ans, j'ai travaillé un petit peu, un an ou deux, des petits contrats aussi... ce n'était pas malhonnête, je n'arrêtais pas de dire que je faisais $200 \$$, on avait le droit de gagner $200 \$ \ldots$ pis ils me surveillaient..." (Caroline, R3)

En somme, les conditions de travail précaires, les imprévus de la vie et l'aide offerte par l'État, qui est souvent conditionnelle et qui place les femmes sous surveillance, génèrent de la souffrance à laquelle elles tendent à répondre par de l'espoir et des stratégies de débrouillardise les aidant à " tenir le coup ». 


\section{Comprendre la disqualification, la stigmatisation et la résistance}

Ainsi, au terme de nos récentes études, nous observons que l'expérience subjective de femmes en situation de pauvreté se manifeste de trois différentes manières, à savoir :

1) être aux prises avec des systèmes qui promeuvent des idéologies dominantes valorisant, entre autres, le succès et la réussite matérielle (Jo, 2012; Luhrmann 2006; O’Brien, 2018) et qui les disqualifient;

2) être stigmatisées, ce qui les mène à éprouver de la honte (Chamberlen, 2017; Jo, 2012; Pollack et Caragata, 2010), et

3) résister aux effets de la pauvreté par l'espoir et la lutte.

L'analyse du discours des femmes met en perspective leur expérience de disqualification. Selon Paugam (2002), cette dernière affecte le statut social et constitue une atteinte à la dignité humaine. En effet, la société néolibérale tend à hiérarchiser les individus selon leur statut économique associé au mérite individuel, disqualifiant ainsi certaines personnes par rapport à d'autres. L'écart entre les attentes sociales en matière de succès et de réussite matérielle et les conditions objectives de pauvreté place ainsi les femmes en position d'échec (Chase et Walker, 2012). Or, la hiérarchisation et la disqualification sociale produites par le néolibéralisme semblent constituer des dimensions à l'origine de la souffrance vécue par les femmes en situation de pauvreté. Cette souffrance peut générer du désespoir, qui à son tour peut conduire à des pensées suicidaires ou encore à des tentatives de suicide (Broussard, Joseph et Thompson, 2012). Qui plus est, bien que la souffrance soit essentiellement une expérience subjective, elle est le fruit d'un contexte social, politique et économique qui la produit. L'analyse du discours des femmes met aussi en perspective leur expérience de stigmatisation et met en lumière des marqueurs qui étiquettent les femmes en situation de pauvreté, les traitant, par exemple, de paresseuses, d'inadéquates et de non méritantes (Savoie, Albert et Lanteigne, 2018). De plus, la pauvreté est génératrice d'une expérience de honte pour les personnes qui y sont confrontées (Chase et Walker, 2012; Jo, 2012; Jovelin, 2017; Savoie, Albert et Lanteigne, 2018). Essentiellement produite par le regard social posé par des individus qui n’ont souvent pas expérimenté la pauvreté (Lister, 2004), c'est sur le plan individuel que la honte se manifeste par l'intériorisation de ce regard (De Gaulejac, 1989). Le regard social est ainsi observable, voire objectif, alors que son intériorisation relève d'une expérience subjective (Jo, 2012). L'aspect objectif de ce regard producteur de honte passe, entre autres, par les institutions et services qui viennent en aide aux personnes en situation de 
pauvreté. En ce sens, que ce soit par la déshumanisation du processus d'aide ou encore par l'attitude de certains intervenants impliqués (traitement homogène, comme un numéro, regard de haut, manque d'écoute, distance culturelle, attitude de jugement), les institutions engendrent de la honte (Savoie, Albert et Lanteigne, 2018). Or, la honte d'être pauvre marque l'expérience personnelle de diverses manières exacerbant ainsi la souffrance ressentie. Ainsi, la honte, entre autres, accentue la marginalisation, contribue à l'exclusion, incite au retrait social, génère un vécu d'ostracisme, atteint à la dignité, à la confiance en soi et à l'estime de soi, et induit un sentiment d'échec parental quand on ne peut offrir aux siens ce que les autres ont (Chase et Walker, 2012; Jo, 2012; Savoie, Albert et Lanteigne, 2016). Par ailleurs, la honte est aussi associée à la fierté, alors que pour se préserver du dur regard des autres (pitié, mépris, etc.), et par souci de sa propre dignité, certaines adopteront des comportements visant à dissimuler leur situation.

De surcroit, la honte se manifeste sur un continuum, variant en fonction de l'intensité et de la durée des émotions qu'elle produit, passant de l'embarras jusqu'à l'humiliation (Scheff, 2011).

Enfin, l'analyse du discours des femmes met en perspective leurs modes d'adaptation et de résistance. Ces modes d'adaptation se traduisent à la fois par l'espoir de s'en sortir et par les luttes menées pour tenir le coup. Or, le rapport à l'espoir n'est pas statique, en ce qu’il varie au gré des expériences du quotidien. Un événement peut être générateur d'espoir, alors qu'un autre peut l'éliminer. Par exemple, le fait d'obtenir un emploi ou encore de retourner aux études en s'inscrivant dans un programme d'alphabétisation peut constituer une source d'espoir pour transformer sa vie (Savoie et Gaudet, 2013). De plus, certaines femmes, pour leur part, retrouvent de l'espoir dans la maternité et dans le sentiment d'utilité que procure le fait de s'occuper des enfants (Lisse, 2007). En revanche, l'avènement d'un problème de santé peut produire du désespoir (Reid et Tom, 2006; Reutter et collab. 2009; Savoie et collab. 2016).

En ce qui a trait à la lutte, Jacquinet (2004) parle de se réaliser autrement et de résister pour tenir bon. Dans le discours des femmes rencontrées, cette résistance se manifeste par l'indignation, la revendication et la lutte contre le système. Par ailleurs, leurs modes d'adaptation passent aussi par des stratégies de survie, dont la gestion financière au quotidien, à savoir « économiser, chercher des aubaines, prioriser les paiements, emprunter, négocier et, de manière récurrente, se priver [...]. Ces modes de débrouillardise qu’elles déploient sont nécessaires dans un contexte d'inégalité de genre, de classe sociale et de ruralité " (Savoie, Albert et Lanteigne, 2016, p. 60). Malgré la souffrance que la pauvreté leur inflige, les femmes rencontrées font appel à l'espoir et leur combativité pour donner sens à sa vie et s'y accrocher. 


\section{Conclusion}

Cet article a voulu mettre l'accent sur l'histoire des femmes rencontrées, dans une intention d'accéder à leur voix, pour dépasser le fait de parler d'elles à la troisième personne de manière à les entendre parler d'elles au « JE ». Il s'est agi de reconnaitre leur souffrance telle qu'elles l'ont exprimée, en allant au-delà de la théorisation qu'il est possible d'en faire. La voix et l'expérience des personnes en situation de pauvreté sont rarement entendues (Lister, 2004). Or, la narration de ces femmes illustre trois dimensions soit la disqualification, la stigmatisation et les modes d'adaptation et de résistance. Au-delà du discours de reproche et de répression qui est souvent dirigé à l'endroit des femmes en situation de pauvreté, la souffrance dont elles témoignent oblige à dépasser une lecture blâmante (poor-blaming) et abaissante (poor-shaming) de leur situation de pauvreté (Barton et Davis, 2018, p. 192), en vue d'une meilleure compréhension nécessaire à la lutte contre la pauvreté. Les rencontres avec ces femmes dans le cadre de ces trois études et leur histoire de vie réitèrent le fait que personne n'est à l'abri de la pauvreté. Or, si personne n'est à l'abri de la pauvreté, et que la pauvreté génère autant de souffrance, comment se fait-il que notre société et ses institutions refusent de reconnaitre cette dernière et continuent de responsabiliser les personnes de leur situation? Il importe pour le travail social de revoir son rapport au pouvoir professionnel dont il est investi de manière à intervenir dans un souci de promotion de la dignité humaine (Gupta, 2015), de reconnaissance et d'actualisation des droits de la personne et de solidarité.

\section{Bibliographie}

ALBERT, Hélène, et collab. (2019). "Quand l'État appauvrit les femmes : réalités de travailleuses de soins à domicile francophones au Nouveau-Brunswick ", Revue canadienne de service social, (à paraître).

BARTON, Alana, et Davis HOWARD (2018). «From empowering the shameful to shaming the empowered: Shifting depictions of the poor in 'reality TV'", Crime Media Culture, Vol.14, № 2, p. 191-211.

BASINSKI, Catherine (2007). « Pauvreté au travail: l'emploi, un rempart pour préserver sa place et sa dignité? ", Pensée plurielle, Vol. 3, № 16, p. 85-99.

BERTAUX, Daniel (2010). L'enquête et ses méthodes. Le récit de vie, $3^{e}$ édition, Paris, Les Éditions Armand Colin, 126 p. 
BROUSSARD, Anne C., Alfred L. JOSEPH et Marco THOMPSON (2012). «Stressors and coping strategies used by single mothers living in poverty ", Affilia: Journal of Women and Social Work, Vol. 27, № 2, p. 190-204.

CHAMBERLEN, Anastasia (2017). "Changing bodies, ambivalent subjectivities, and women's punishment ", Feminist Criminology, Vol. 12, № 2, p. 125-144.

CHASE, Elaine, et Robert WALKER (2012). "The Co-construction of shame in the context of poverty: Beyond a threat to the social bond ", Sociology, Vol. 47, No 4, p. 739-754.

COALITION POUR L'ÉQUITÉ SALARIALE DU NOUVEAU-BRUNSWICK (2016). Accroitre la prospérité économique des femmes dans le secteur de prestations de soins, réf. du 12 mai 2019, http://www.equite-equity.com/userfiles/file/Needs\%20Assessment_fr.pdf

De GAULEJAC, Vincent (1989). «Honte et pauvreté ", Santé mentale au Québec, Vol. 14, No 2, p. 128-137.

GOUVERNEMENT DU NOUVEAU-BRUNSWICK (2016). Profil égalité, réf. du 12 mai 2019, https://www2.gnb.ca/content/dam/gnb/Departments/eco-bce/WEB-EDF/pdf/fr/Equality\%20 Profile\%202016-PDF-F.pdf

GRELL, Paul (2015). Adolescence et suicide, Paris, Berg International Éditeurs, 216 p.

GUPTA, Anna (2015). « Poverty and shame - message for social work », Critical and Radical Social Work, Vol. 3, No 4, p. 131-139.

JACQUINET, Serge (2004). "Souffrance sociale des usagers et malaise des travailleurs sociaux ", Pensée plurielle, Vol. 2, № 8, p. 39-49.

JO, Yongmie Nicola (2012). «Psycho-social dimensions of poverty: When poverty becomes shameful ", Critical Social Policy, Vol. 33, №3, p. 514-531.

JOVELIN, Emmanuel (2017). « La honte des pauvres : l'exemple des femmes SDF », Pensée plurielle, Vol. 1, № 44, p. 73-83.

KAUFMANN, Jean-Claude (2006). L'enquête et ses méthodes. L'entretien compréhensif, Paris, Les Éditions Armand Colin, 128 p.

KLEIN, Juan-Luis, et Christine CHAMPAGNE (2011). « La lutte contre la pauvreté et l'exclusion sociale. Approches et stratégies institutionnelles ", dans Juan-Luis Klein et Christine Champagne (dirs.), Initiatives locales et lutte contre la pauvreté et l'exclusion, Québec, Les Presses de l'Université du Québec, p. 31-44.

KRUMER-NEVO, Michal (2005). « Reading a poor woman's life: Issues and dilemmas », Afflia:Journal of Women and Social Work, Vol. 20, № 1, p. 87-102.

LAMOUREUX, Diane (2016). Les possibles du féminisme. Agir sans "nous", Montréal, Éditions du Remue-Ménage, $280 \mathrm{p}$. 
LISSE, Élisabeth (2007). " Monoparentalité et sociabilité féminine. Apprentissages du rôle de mère en cité populaire ", Ethnologie française, Vol. 37, No 4, p. 733-741.

LISTER, Ruth (2004). Poverty, Cambridge, Policy Press, 238 p.

LUHRMANN, Tanya Mario (2006). "Subjectivity », Anthropological Theory, Vol. 6, No 3, p. 345-361.

NOVAK, Tony (1995). "Rethinking poverty», Critical Social Policy, Vol. 15, No 44-45, p. 58-74.

O'BRIEN, Anne (2018). "(Not) getting the credit: women, liminal subjectivity and resisting neoliberalism in documentary production ", Media, Culture \& Society, Vol. 40, № 5, p. 673-688.

OLLIVIER, Michèle, et Manon TREMBLAY (2000). Questionnements féministes et méthodologie de la recherche, Paris, Les Éditions l'Harmattan, 256 p.

PAILLÉ, Pierre, et Alex MUCCHIELLI (2012). L'analyse qualitative en sciences humaines et sociales, $3^{\mathrm{e}}$ édition, Paris, Les Éditions Armand Colin, $251 \mathrm{p}$.

PAUGAM, Serge (2002). La disqualification sociale, Paris, Presses Universitaires de France, 256 p.

POLLACK, Shoshana, et Lea CARAGATA (2010). "Contestation and accommodation: Constructions of lone mothers' subjectivity through workfare discourse and practice », Affilia: Journal of Women and Social Work, Vol. 25, No 3, p. 264-277.

REID, Colleen, et Robin A. LEDREW (2013). "The burden of being 'employable': Underpaid and unpaid work and women's health ", Affilia: Journal of Women and Social Work, Vol. 28, № 1, p. 79-93.

REID, Colleen, et Allison TOM (2008). " Poor women's discourses of legitimacy, poverty, and health ", Gender and Society, Vol. 20, № 3, p. 402-421.

REUTTER, Linda I., et collab. (2009). "Who do they think we are, anyway?": Perceptions of and responses to poverty stigma ", Qualitative Health Research, Vol. 19, No 3, p. 297-311.

ROSE, Ruth (2014). L'équité salariale dans les services de proximité au Nouveau-Brunswick, réf. du 12 mai 2019, http://www.equite-equity.com/userfiles/file/2015-10-18_RAPPORT_Ruth\%20Rose.pdf

SAVOIE, Lise, Hélène ALBERT et Isabel LANTEIGNE (2018). " Mythes et mensonges sur les femmes en situation de pauvreté en milieu rural : Lever le voile sur des réalités de femmes sujets de leur vie ", Nouvelles pratiques sociales, Vol. 29, № 2.

SAVOIE, Lise, Hélène ALBERT et Isabel LANTEIGNE (2016). «Un filet de sécurité sociale troué : la métaphore de la courtepointe pour raconter des histoires de femmes débrouillardes vivant en situation de pauvreté et qui doivent composer avec une santé fragile ", Reflets : revue d'intervention sociale et communautaire, Vol. 22, № 2, p. 44-68.

SAVOIE, Lise, et Jeanne d'Arc GAUDET (2013). "Réussir son insertion professionnelle: une analyse féministe de trajectoires sociales de femmes qui se sont engagées dans un processus d'alphabétisation ", Recherches féministes, Vol. 26, № 1, p. 31-48. 
SAVOIE, Lise, et collab. (2016). « Des femmes en situation de pauvreté : prendre en charge sa santé en contexte de ruralité ", Intervention, $\mathrm{N}^{\circ} .143$, p. 15-31.

SCHEFF, Thomas J. (2011). "Shame in self and society ", Symbolic Interaction, Vol. 26, № 2, p. 239-262.

YUVAL-DAVIS, Nira (2006). "Intersectionality and feminist politics », European Journal of Women's Studies, Vol.3, No 2, p.193-209. 